\title{
Dispensación de fentanilo y tapentadol en farmacia comunitaria: perfil del paciente e indicación terapéutica en dolor crónico no oncológico
}

\author{
Alicia González Rodríguez ${ }^{1,3}$, Daniel Reyes Estévez ${ }^{1,3}$, Eloi Merencio Naudin ${ }^{2,3}, \mathrm{M}^{a}$ Edelmira Córcoles Ferrándiz ${ }^{1,3}$ \\ 1. Grupo de Dolor de SEFAC. 2. Coordinador del Grupo de Dolor de SEFAC. 3. Farmacéutico/a comunitario/a.
}

\section{PALABRAS CLAVE}

Analgésicos opioides, dolor crónico, cumplimiento y adherencia al tratamiento, farmacia comunitaria, farmacéutico comunitario, servicios profesionales farmacéuticos

\section{ABREVIATURAS}

FC: farmacéuticos comunitarios DCNO: dolor crónico no oncológico EVA: escala visual analógica SNC: sistema nervioso central AEMPS: Agencia Española de Medicamentos y Productos Sanitarios OMS: Organización Mundial de la Salud

PRM: problemas relacionados con el medicamento

RNM: resultados negativos de la medicación

SEFAC: Sociedad Española de Farmacia Familiar y Comunitaria. PNT: procedimiento normalizado de trabajo

CRD: cuaderno de recogida de datos $\mathrm{AF}$ : atención farmacéutica

\section{RESUMEN}

Introducción: Durante los últimos años ha existido un aumento en la prescripción y dispensación de opioides fuertes, destacando fentanilo y tapentadol. La elevada prevalencia de pacientes con dolor crónico no oncológico, que acuden a las farmacias comunitarias solicitando su dispensación, justifica el interés profesional.

Objetivos: Conocer la indicación de dichos opioides fuertes en la muestra.

Conocer el perfil de los pacientes que utilizan dichos tratamientos en dolor no oncológico.

Material/Métodos: Estudio observacional, descriptivo, transversal realizado por 139 farmacéuticos comunitarios. Población diana: mayores de 18 años que acuden a la farmacia comunitaria con prescripción de fentanilo o tapentadol. Las variables recogidas fueron edad, sexo, patología, carácter crónico/agudo, indicación, motivo del dolor, principios activos dispensados (conocimiento de posología, administración, posibles reacciones adversas), adherencia (Test de Haynes-Sackett), dolor (Escala Visual Analógica) e intervención del farmacéutico.

Resultados: La muestra fue constituida por 559 pacientes. Un 85,5\% tenía indicación no oncológica, de los cuales 4,0\% fueron indicados para tratamientos agudos y $96,0 \%$ indicados para dolor crónico no oncológico. De las indicaciones no oncológicas el 20,3\% fue para lumbalgias, el 29,1\% para artrosis, y el 50,6\% para otras patologías.

Con indicación no oncológica un 73,0\% fueron mujeres y 54,9\% mayores de 70 años.

Conclusiones: La utilización de fentanilo y tapentadol es más frecuente en mujeres y en pacientes mayores de 70 años.

Los opioides fuertes fentanilo y tapentadol dispensados en farmacia comunitaria se utilizan principalmente para tratar dolor crónico no oncológico. Las patologías no oncológicas tratadas mayormente con los opioides fuertes fentanilo y tapentadol son artrosis y lumbalgias.

Dispensing of fentanyl and tapentadol in community pharmacy: Patient profile and therapeutic indication in chronic non-oncological pain

\section{ABSTRACT}

Introduction: In recent years there has been an increase in the prescription and dispensation of strong opioids, highlighting fentanyl and tapentadol. The professional interest has been justified by the high prevalence of patients with chronic non-oncological pain that come to the community pharmacy.

Objectives: Knowing the indication of the named strong opioids in the sample.

The profile recognition of patients that use those treatments with non-oncological pain.

Material/Methods: Observational, descriptive and transverse study performed by 139 community pharmacists. Target population: people over 18 years old that come to community pharmacy with a prescription of fentanyl or tapentadol. The collected variables were age, sex, pathology, chronic/acute character, indication, reason of pain, dispensed active principles (knowledge of posology, administration, possible adverse reactions), adherence (Haynes-Sackett Test), pain (Visual Analogic Scale), and the pharmacist's intervention. 


\section{KEYWORDS}

Analgesics opioid, chronic pain, treatment adherence and compliance, community pharmacy, community pharmacist, pharmaceutical services
Results: 559 patients were included in the research. 85,5\% had non-oncological indication of which 4,0\% were indicated for acute treatments and 96,0\% for chronic non-oncological pain. Patients who had non-oncological indication: 20,3\% low back pain, 29,1\% osteoarthritis, and 50,6\% other pathologies.

Patients with non-oncological pain $73,0 \%$ were women and 54,9\% were patients over 70 years old.

Conclusions: Use of fentanyl and tapentadol is more common in women and in patients over 70 years old.

The strong opioids fentanyl and tapentadol dispensed in Community Pharmacy are used to treat mainly non-oncological pain. The most non-oncological pathologies treated with fentanyl and tapentadol are osteoarthritis and low back pain.

\section{Introducción}

El dolor constituye una patología de importancia creciente a nivel mundial (1). Su prevalencia aumenta de forma paralela al envejecimiento de la población y cronificación de las enfermedades.

Las cifras de prevalencia de dolor crónico en la población en general se encuentran entre el 15\% y el $25 \%$, según datos basados en la población de Estados Unidos (2), cifra que aumenta al 50\% en los mayores de 65 años y en cualquier grupo afecta más a las mujeres que a los hombres. En 2011, el Ministerio de Sanidad editó una publicación denominada "Unidad de tratamiento del dolor. Estándares y recomendaciones de calidad y seguridad". En ella, se recopilaron todos los estudios de prevalencia del dolor crónico desde el año 2001 (3), reflejando una prevalencia para el dolor crónico del 23,8\%, además de, una relación mujer/hombre de $37 / 20$.

Por otro lado, la última Encuesta Nacional de Salud (4) en España (2011-2012) muestra que uno de cada seis adultos encuestados refiere dolor lumbar/cervical crónico o artrosis, dolencias referidas como las principales causas de dolor crónico no oncológico (DCNO) $(5,6)$.

\section{Justificación}

Dentro del dolor crónico, el DCNO supone un reto debido a su complejidad e imprevisibilidad de respuesta al tratamiento. Actualmente, aunque existe amplia bibliografía que recomienda el uso tanto en dolores nociceptivos (7) como neuropáticos, solo existe amplia experiencia clínica en dolor nociceptivo, ya que en dolor neuropático existe una gran variabilidad de tipos de dolor.
La efectividad a largo plazo de los opioides fuertes utilizados en el DCNO sigue siendo controvertida, ya que la mayoría de los estudios que hablan sobre su efectividad, no son superiores a 12 semanas de duración y los estudios sobre su seguridad no van más allá de 1 año (8), por lo que tienen que ser indicados y prescritos con precaución y siempre valorando a cada paciente de forma individual (9).

La efectividad inicial puede desaparecer aun siendo los pacientes adherentes al tratamiento. Al no tener techo de dosis, altas dosificaciones pueden originar efectos adversos propios de su uso y posología. Afectan al Sistema Nervioso Central (SNC) y el aparato gastrointestinal (náuseas, vómitos, estreñimiento, mareos, somnolencia y sedación).

En los últimos años, ha habido un aumento en la prescripción y, como consecuencia, un crecimiento en la dispensación de fármacos opioides fuertes. Fentanilo y tapentadol son un ejemplo de ese aumento (10). Tanto es que desde la Administración se están realizando programas de inspección para valorar el aumento de consumo de fentanilo.

En 2017 la Agencia Española de Medicamentos y Productos Sanitarios (AEMPS) emitió un informe "Utilización de medicamentos opioides en España durante el periodo 2008-2015" (11), donde se puede ver el aumento de consumo de todos los grupos de opioides, y tanto fentanilo como tapentadol tienen un aumento exponencial.

Cada vez se utiliza más este último escalón de la escalera analgésica clásica de la Organización Mundial de la Salud (OMS) para tratar dolor crónico, ya sea de origen oncológico o no oncológico (11).
En este entorno de práctica de la atención farmacéutica el farmacéutico comunitario puede implicarse aún más evaluando la efectividad de los tratamientos y solucionando los problemas relacionados con el medicamento (PRM) y resultados negativos de la medicación (RNM), que identifique en su práctica profesional. En patologías crónicas que cursan con dolor crónico resulta conveniente que el farmacéutico comunitario pueda evaluar la respuesta terapéutica a los tratamientos en estos pacientes, $\mathrm{y}$ para esta valoración se tiene que tener en cuenta el grado de dolor.

La medición objetiva del dolor es un proceso complejo, resulta difícil estimar, medir o valorar el dolor que siente una persona. Sin embargo, su cuantificación es muy importante y su evaluación es una actividad clave derivada de una intervención informativa y educativa para ayudar a mejorar la percepción que el paciente tiene sobre su dolor (12).

La alta prevalencia de los pacientes aquejados de DCNO, que acuden a las farmacias comunitarias, justifica el interés profesional por ampliar conocimientos sobre el dolor crónico y los tratamientos efectivos, para poder abordar a estos pacientes adecuadamente. Por ello, el farmacéutico comunitario tiene un papel muy importante en la dispensación de estos medicamentos, garantizando un uso responsable.

Ante ello, y habiendo observado dificultades en el uso de los opioides fuertes, planteamos un amplio estudio en la farmacia comunitaria para conocer la indicación de los opioides fuertes fentanilo y tapentadol en la población diana, el perfil de los pacientes tratados con estos opioides fuertes para DCNO, evaluar la adherencia de estos pacientes con dolor 
y conocer las causas de falta de adherencia y dificultades en su uso, así como evaluar la intensidad del dolor.

El trabajo que aquí se presenta analiza el perfil del paciente que utiliza estos analgésicos opioides, así como para qué tipo de dolor crónico no oncológico, indicación terapéutica, han sido prescritos, y tiene como objetivos:

- Analizar el número de prescripciones de fentanilo y tapentadol con indicación de dolor oncológico y no oncológico.

- Cuantificar el número de dichas prescripciones que son para dolor agudo y dolor crónico con indicación no oncológica.

- Conocer la indicación de dichos opioides fuertes en la muestra.

- Conocer el perfil de los pacientes que utilizan opioides fuertes en DCNO.

- Conocer quién retira la medicación de la farmacia comunitaria: pacientes o cuidadores.

\section{Material y método Diseño del estudio}

Estudio de corte descriptivo, observacional, transversal, multicéntrico y de ámbito nacional, realizado entre enero y marzo de 2018 por 139 farmacéuticos comunitarios españoles, e impulsado por el grupo de Dolor de la Sociedad Española de Farmacia Familiar y Comunitaria (SEFAC).

\section{Sujetos}

Población diana: mayores de 18 años que llegan a la farmacia comunitaria con prescripción de fentanilo o tapentadol.

\section{Criterios de inclusión}

Pacientes mayores de 18 años que aceptan participar y dan su consentimiento informado escrito.

\section{Criterios de exclusión}

Se excluyeron del estudio los pacientes menores de 18 años, los pacientes con dificultad de comunicación y aquellos que no aceptaron participar.

\section{Procedimiento}

A través de la plataforma online www.investigacionsefac.org se faci- litó a los farmacéuticos participantes un procedimiento normalizado de trabajo (PNT): PNT Estudio Análisis del uso de fentanilo y tapentadol en farmacia comunitaria (anexo 1), con el objetivo de servir como guia a todos los farmacéuticos participantes. También se pretendía recordar algunas cuestiones intrínsecas a la dispensación de los fármacos que contenían los principios activos fentanilo o tapentadol en relación con las recomendaciones con respecto al uso adecuado de estos medicamentos Principios activos y formas de liberación (anexo 2) y facilitar normas de educación sanitaria en pacientes no oncológicos Hábitos de vida saludable para el paciente con dolor crónico no oncológico (anexo 3).

Para facilitar su desarrollo, así como una normalización entre todos los investigadores a la hora de la recogida de datos, se proporcionó también a los farmacéuticos participantes a través de la plataforma online www.investigacionsefac.org el cuaderno de recogida de datos (eCRD): Registro de dispensación de opioides fuertes para dolor no oncológico (figura 1), específicamente elaborado para el estudio.

Finalmente, a modo de entrenamiento se ofreció a los participantes un vídeo de aprendizaje para la toma de datos.

Durante el periodo del estudio (enero a marzo del 2018), los farmacéuticos comunitarios cumplimentaron un e-CRD para cada paciente que permitió recoger información sobre el paciente, edad, sexo, patología, tiempo de evolución, indicación, motivo del dolor y principios activos dispensados. Además, se registraron otras variables para el estudio completo: conocimiento de posología, administración y posibles reacciones adversas, evaluación de adherencia (Test de Haynes-Sackett), dolor (Escala Visual Analógica -EVA), expectativas relacionadas al tratamiento y la intervención del farmacéutico.

\section{Análisis de datos}

Una vez finalizado el período del estudio, se llevó a cabo el registro informático de los datos recogidos de todos los pacientes y se procedió al análisis de los mismos. El tratamiento estadístico de los datos se realizó con una hoja de aplicación informática MSExcel ${ }^{\circledR}$

Las variables cuantitativas se expresaron mediante las medidas de tendencia central y de dispersión (media y desviación estándar) y las variables cualitativas como frecuencias absolutas y relativas, incluyendo el intervalo de confianza al 95\%. Se utilizaron los test de la Chi cuadrado para las comparaciones de variables cualitativas y t de Student en las cuantitativas. Se consideró significativa una $\mathrm{p}<0,05$.

\section{Resultados}

Participaron 139 FC de todas las comunidades autónomas de España. 566 pacientes solicitaron una dispensación de fentanilo o tapentadol, de ellos 559 (98,8\%) aceptaron participar en el estudio y $7(1,2 \%)$ lo rechazaron.

De los participantes, 173 (30,9\%) fueron hombres y $386(69,1 \%)$ fueron mujeres. 307 (54,9\%) fueron mayores de 70 años, 234 (41,9\%) tenían entre 40 y 70 años y 18 (3,2\%) estaban entre 18 y 40 años.

Fentanilo fue dispensado en 318 $(56,9 \%)$ casos y tapentadol en 241 $(43,1 \%)$.

La dispensación de estos medicamentos fue diferente en función del sexo y la edad del paciente, siendo significativo en el caso de la edad (tabla 1).

La prescripción de estos opiáceos fue mayoritariamente para dolor no oncológico, fueron 478 pacientes (85,5\% IC95\% 82,6\% - 88,4\%), y dolor oncológico fueron 81 pacientes (14,5\% IC95\% 11,6\% - 17,4\%). En la figura 2 se indica la distribución de tipo de indicación en función del sexo. Las diferencias son significativas $(\mathrm{p}=0,0000009)$.

En dolor no oncológico el 23,0\% fueron hombre y el 73,0\% fueron mujeres. En la figura 3 se indica el perfil del paciente, sexo y edad, en la prescripción de dolor no oncológico.

Hubo diferencias significativas $(p=0,0007)$ en cuanto a la prescripción de cada principio activo, fentanilo o tapentadol, según el tipo de indicación. Se indican en la tabla 2.

Dentro de las prescripciones de dolor no oncológico, 459 (96,0\% IC95\% $94,3 \%$ - 97,8\%) fueron para dolor crónico, y 19 (4,0\% IC95\% 2,2\% - 5,7\%) para dolor agudo. En hombres la proporción de dolor agudo fue ligeramente mayor pero las diferencias no fueron significativas $(p=0,32)$.

Las patologías más frecuentes en DCNO fueron lumbalgia $\mathrm{y}$ artrosis con 97 y 139 casos respectivamente. 


\section{Registro de dispensación de estupefacientes para dolor no oncológico}

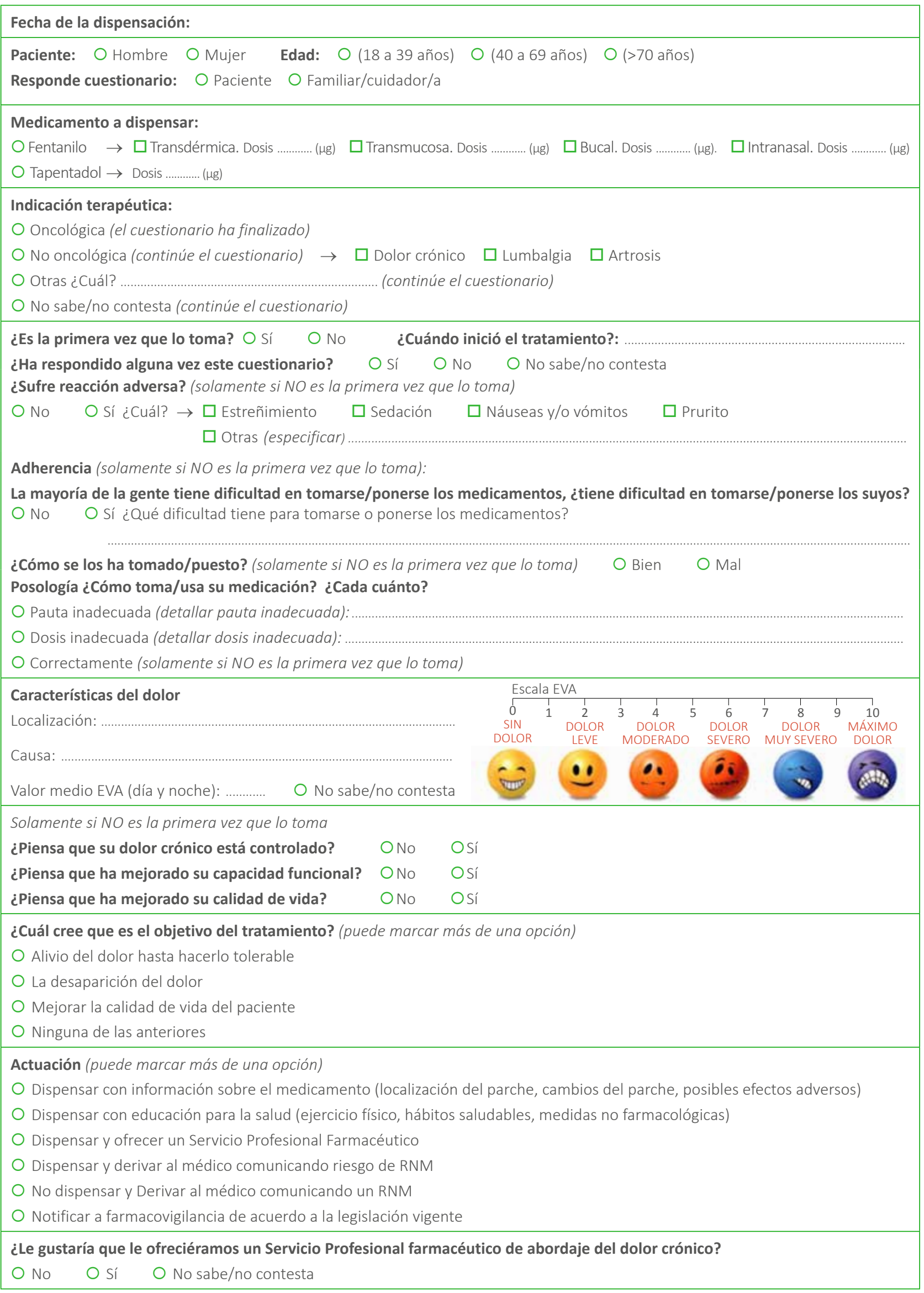

Figura 1 Formulario de recogida de datos 
Tabla 1 Medicamento dispensado según el sexo y la edad del paciente

\begin{tabular}{|l|c|c|c|c|c|c|}
\hline & Fentanilo & Tapentadol & Total & Fentanilo & Tapentadol & Total \\
\hline Hombre & 109 & 64 & 173 & $63 \%$ & $37 \%$ & $100 \%$ \\
\hline Mujer & 209 & 177 & 386 & $54 \%$ & $46 \%$ & $100 \%$ \\
\hline Total & 318 & 241 & 559 & & $p=0,0505$ & \\
\hline (18 a 39 años) & 9 & 9 & 18 & $50 \%$ & $50 \%$ & $100 \%$ \\
\hline (40 a 69 años) & 115 & 119 & 234 & $49 \%$ & $51 \%$ & $100 \%$ \\
\hline (>70 años) & 194 & 113 & 307 & $63 \%$ & $37 \%$ & $100 \%$ \\
\hline Total & 318 & 241 & 559 & & $p=0,0040$ & \\
\hline
\end{tabular}

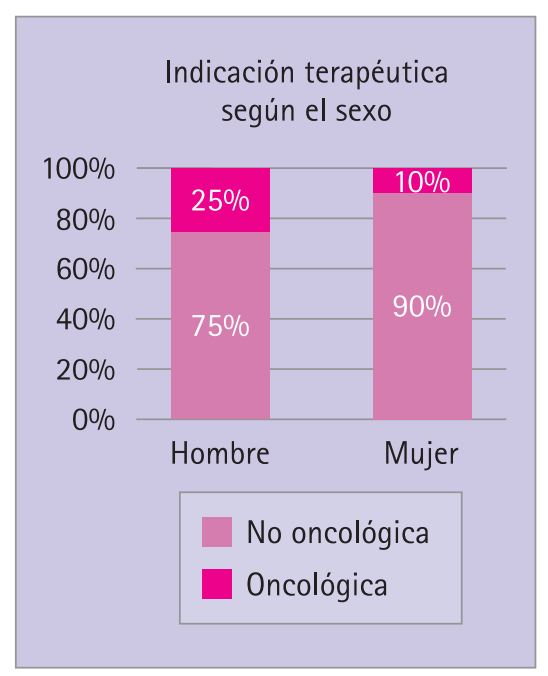

Figura 2 Indicación terapéutica según el sexo del paciente

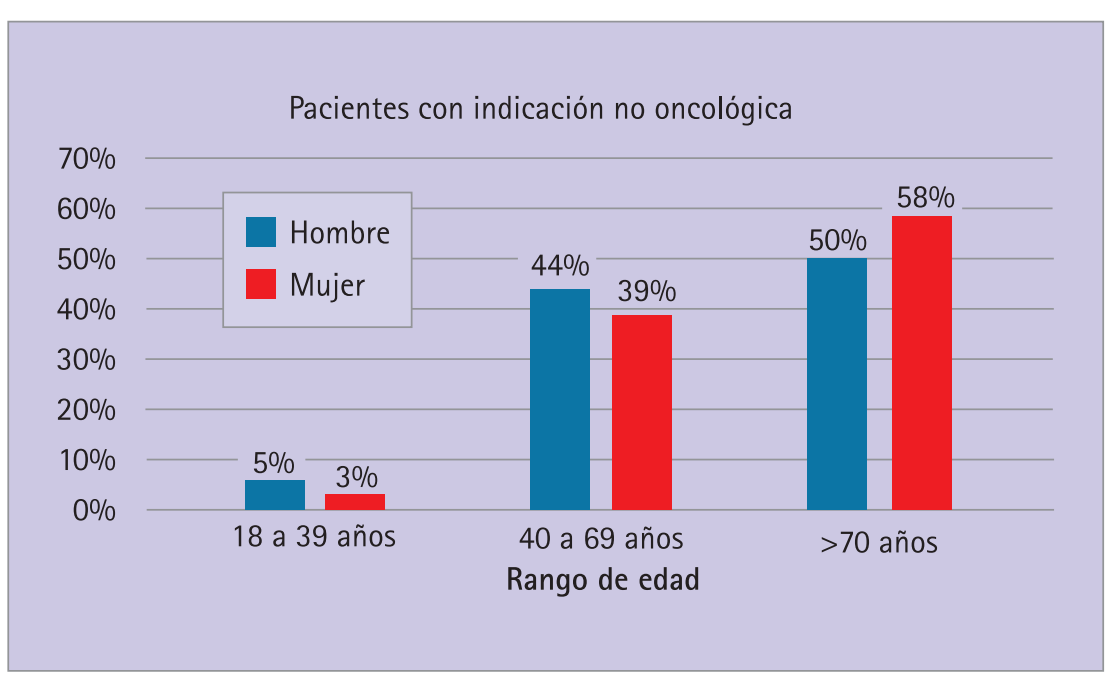

Figura 3 Perfil del paciente con DCNO

Tabla 2 Indicación terapéutica según el medicamento dispensado

\begin{tabular}{|l|c|c|c|c|c|c|}
\hline Indicación terapéutica & Fentanilo & Tapentadol & Total & Fentanilo & Tapentadol & Total \\
\hline No oncológica & 258 & 220 & 478 & $81 \%$ & $91 \%$ & $86 \%$ \\
\hline Oncológica & 60 & 21 & 81 & $19 \%$ & $9 \%$ & $14 \%$ \\
\hline Total & 318 & 241 & 559 & $100 \%$ & $100 \%$ & $100 \%$ \\
\hline
\end{tabular}

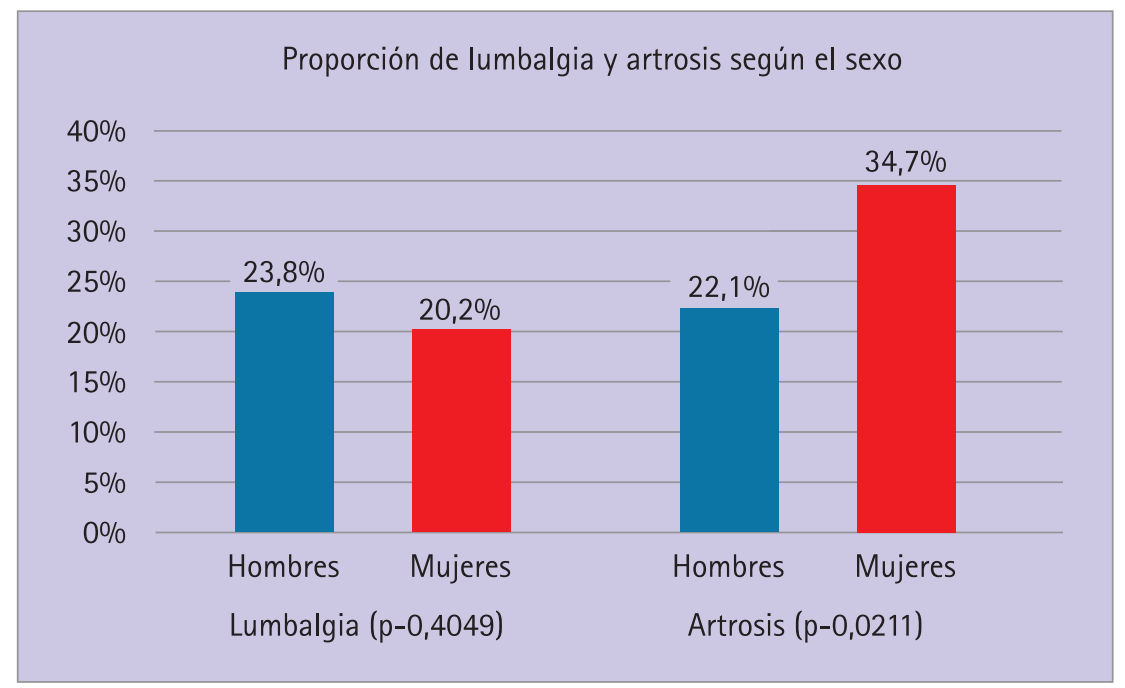

Figura 4 Proporción de hombres y mujeres con prescripción para lumbalgia y artrosis
Suponen el 20,3\% y el 29,1\% del total de prescripciones para DCNO.

La prescripción para lumbalgia $\mathrm{y}$ artrosis en función del sexo puede verse en la figura 4 hubo diferencias significativas para la artrosis.

Tapentadol se prescribió más para la lumbalgia y fentanilo para la artrosis. Los datos pueden verse en la figura 5. Hubo diferencias significativas.

En los casos de dolor no oncológico es el paciente el que acude a la farmacia y responde el cuestionario, en el 63,4\% de los casos, y el cuidador en los restantes. Pero si se trata de dolor oncológico el paciente solo acude en el 44,4\% de los casos. 


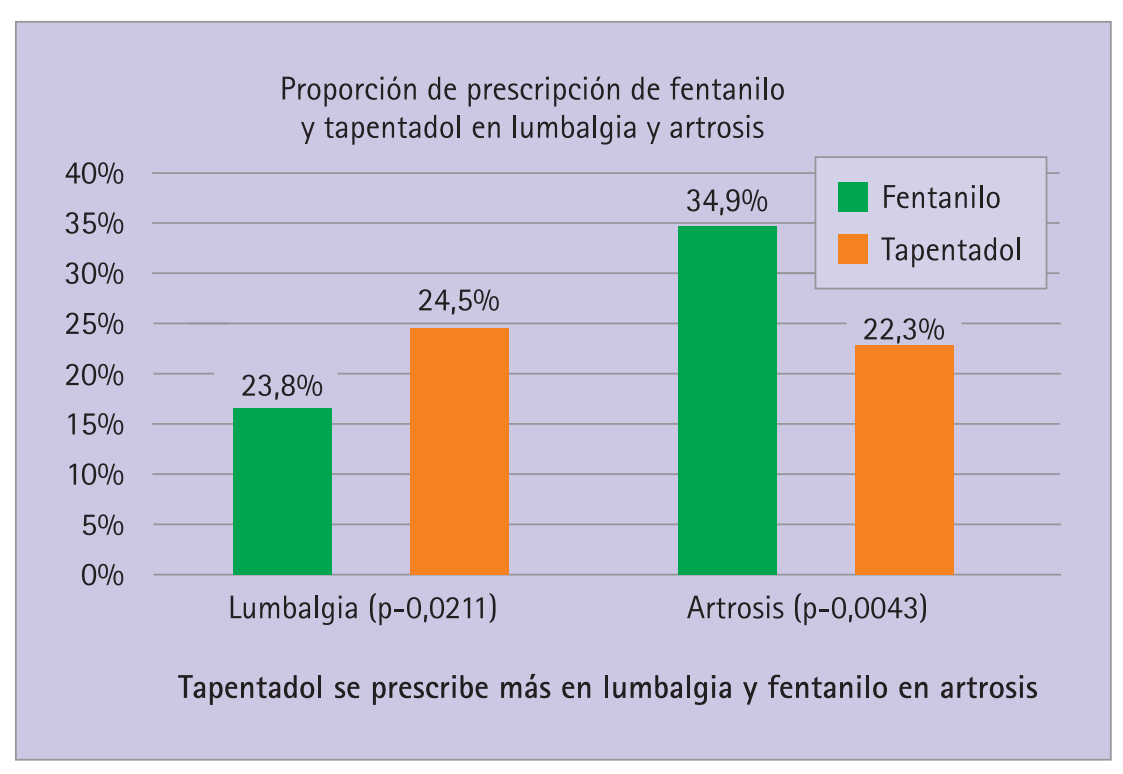

Figura 5 Opiáceos prescritos para los problemas de salud más frecuentes

\section{Discusión}

En nuestro estudio realizado sobre el análisis del uso de fentanilo y tapentadol en farmacia comunitaria se puede observar que el $86 \%$ de los pacientes presentaron una indicación no oncológica, de los cuales el 96\% fue por DCNO y solo 4\% para dolor agudo no oncológico.

Nuestros datos reflejan una mayor utilización de opioides fuertes para tratar el DCNO frente a la indicación oncológica que supuso un 14\%. Una explicación podemos encontrarla en el análisis que hace el Ministerio de Sanidad (7), donde se expone que el uso de opioides mayores ha experimentado un notable aumento, en gran medida por el incremento de uso para dolor crónico no oncológico.

Observamos también diferencias según sea el sexo del paciente, siendo mayor el número de mujeres que de hombres y en las mujeres es superior el porcentaje de indicación no oncológica respecto de los varones, en mujeres es más frecuente la indicación no oncológica ( $\mathrm{p}<0,0001)$. Nuestros datos coinciden con las cifras de mayor prevalencia del DCNO en las mujeres, como se recoge en la publicación del Ministerio de Sanidad donde se recopilan todos los estudios de prevalencia del dolor crónico desde el año 2001 (3).

También se observan diferencias en cuanto al medicamento que se emplea según el sexo. Fentanilo se prescribe más a hombres que a mujeres, mientras que a la inversa tapen- tadol se prescribe más a mujeres que a hombres, aunque las diferencias no llegan a ser significativas ( $p=0,0505)$ como se puede ver en la tabla 1 .

Se han encontrado diferencias según el tipo de opioide fuerte prescrito en relación al tipo de indicación. Se observa que para dolor oncológico se prescribe más fentanilo que tapentadol: $(p \approx 0)$ como se puede ver en la tabla 2 .

En el estudio Ítaca (13), que fue realizado en 100 unidades de dolor de toda España, a 907 pacientes, la mayoría de los pacientes presentaba lumbalgia (52,92\%), mientras que en nuestro estudio las indicaciones para tratamiento del DCNO más relevantes son la artrosis seguida de la lumbalgia.

Fentanilo se prescribe más para artrosis, mientras que tapentadol se prescribe más para lumbalgia $(\mathrm{p}=0,001)$.

Con respecto a las patologías causantes del DCNO también existen diferencias en cuanto al sexo del paciente, las mujeres tienen más artrosis y los hombres más lumbalgia, pero no es significativo $(p=0,062)$ comparando lumbalgia y artrosis. Estas diferencias entre hombres y mujeres pueden deberse a que los hombres reciben menos tratamientos analgésicos, como se analiza en el estudio de Noceda et al (14), en el que se encontraron diferencias significativas en cuanto al sexo, identificándose un mayor número de varones que de mujeres que se encontraban sin tra- tamiento analgésico alguno o que lo recibían en dosis inferiores a las dosis diaria definida. Esta situación puede ser debida tanto al mal cumplimiento por parte del paciente como a la baja prescripción por parte de su médico, o a ambos. Además, según el estudio EPIDOR (15) la mitad de los pacientes no tiene suficiente confianza en su tratamiento farmacológico para el reuma o no está satisfecho con el tratamiento que recibe para el dolor. De acuerdo con la literatura científica, hay más varones que mujeres en esta situación (16). Según el análisis de Noceda et al una explicación es que las mujeres reciben más analgésicos, tal vez debido a la mayor influencia del dolor en su salud física y mental (14).

En nuestro estudio son los mayores de 70 años los que más prescripciones tienen de estos opioides, con un 55\%, seguido de los pacientes de 40 a 69 años con el 42\%. Tras analizar el rango de edad con el medicamento que utiliza el paciente se desprende, sin tener en cuenta a los más jóvenes, que fentanilo se usa más en mayores de 70 años. ( $p=0,001)$, como se puede ver en la tabla 1 .

En los casos de dolor oncológico en más de la mitad de los casos son los familiares/cuidadores los que acudieron a las farmacias comunitarias, mientras que para dolor no oncológico son los propios pacientes, en su mayoría, los que acudieron a las farmacias comunitarias solicitando el tratamiento prescrito, aunque también es considerable el número de familiares/cuidadores que recogen el tratamiento dispensado para el paciente con DCNO. Esta situación nos permite analizar la necesidad de una atención farmacéutica domiciliaria prestada por los FC.

Las limitaciones de nuestro trabajo son el tamaño muestral comparado con el elevado número de prescripciones de fentanilo y tapentadol. En los últimos años ha habido un aumento en la prescripción y dispensación de fármacos opioides fuertes. Fentanilo y el tapentadol son un ejemplo de ese aumento (10).

La alta prevalencia de los pacientes aquejados de DCNO, que acuden a las farmacias comunitarias, justifica el interés profesional por ampliar conocimientos sobre el dolor crónico y los tratamientos efectivos, para poder abordar a estos 
pacientes adecuadamente. Por ello, el farmacéutico comunitario tiene un papel muy importante en la dispensación de estos medicamentos, garantizando un uso responsable de los mismos.

Es recomendable que los FC enseñemos a los pacientes cómo utilizar los opioides fuertes, siendo la no correcta administración uno de los errores que provoca eventos adversos (7). Para ello, en nuestro estudio se ofreció a los pacientes información del uso adecuado de estos tratamientos para disminuir las dificultades que tienen en el uso de estos tratamientos. El principal problema que existe fue ser mayor con problemas de dependencia, quien necesita ayuda que en ocasiones no tiene; otro de los motivos fue la dificultad en la colocación y problemas en la adhesión del parche de fentanilo.

\section{Conclusión}

Los opioides fuertes fentanilo y tapentadol que son dispensados en farmacia comunitaria se utilizan principalmente para tratar DCNO.

Las patologías no oncológicas tratadas mayormente con estos opioides fuertes son artrosis y lumbalgias.

Aunque no resulta significativo, en nuestro estudio las mujeres padecen más artrosis y los hombres más lumbalgias.

La prescripción de fentanilo y tapentadol es mayor en mujeres, y en edades superiores a 70 años, sin distinción de sexo.

La indicación terapéutica de estos opioides fuertes para dolor oncológico es mayor en hombres.

El propio paciente es el que retira en mayor medida estos fármacos en la farmacia comunitaria.

\section{Agradecimientos}

A los 139 farmacéuticos que han colaborado de forma desinteresada en el estudio.
A SEFAC por poner a disposición del grupo de Dolor las plataformas informáticas para llevar a cabo el estudio.

\section{Referencias bibliográficas}

1. López Pérez FJ, Mínguez Martí A, Vicario Sánchez E, Pastor Clérigues A, Sanfeliu García J, Ortega García MP. Experiencia clínica en cambio de opioides en el tratamiento del dolor crónico no oncológico. Farm Hosp (Toledo) 2014; 38(5):411-417.

2. Andrés J, Acuña JP, Olivares A. Dolor en el paciente de la tercera edad. Rev Med Clin Condes 2014; 25(4): 674-686. doi:10.1016/S0716-8640(14)70089-6

3. Palanca Sánchez I, Puig Riera de Conías MM, Elola Somoza I, Bernal Sobrino JL, Paniagua Caparrós JL. G. Unidad de tratamiento de dolor, estándares y recomendaciones [Monografía en Internet]. Madrid: Ministerio de Sanidad, Politica Social e Igualdad; 2011 [acceso 6 de septiembre de 2018] Disponible en: https://es.calameo. com/read/000072525c316dfec325a

4. Encuesta Nacional de Salud 20112012 [Monografía en Internet]. Madrid: Ministerio de Sanidad; 2013 [acceso 4 de septiembre de 2018] Disponible en: http://www.ine.es/prensa/ np770.pdf

5. Dobscha SK, Morasco BJ, Duckart JP, Macey T, Deyo RA. Correlates of prescription opioid initiation and long-term opioid use in veterans with persistent pain. Clin J Pain [revista en internet]. 2013 [acceso 5 septiembre de 2018]; 29(2):102-8. Disponible en: https://www.ncbi.nlm.nih.gov/pubmed/23269280

6. Reid MC, Henderson CR Jr, Papaleontiou M, Amanfo L, Olkhovskaya Y, Moore AA, et al. Characteristics of older adults receiving opioids in primary care: Treatment duration and outcomes. Pain Med 2010; 11(7):1063-71. doi:10.1111/j.1526-4637.2010.00883.x

7. Prácticas seguras para el uso de opioides en pacientes con dolor crónico [Monografía en Internet]. Madrid: Ministerio de Sanidad, Servicios Sociales e Igualdad; 2015 [acceso 2 de septiembre de 2018] Disponible en: https://www.sedolor.es/grupos-de-trabajo/opioides/
8. Reuben DB, Alvanzo A, Ashikaga T, Bogat GA, Callahan CM, Ruffing V, et al. National Institutes of Health Pathways to Prevention Workshop: The Role of Opioids in the Treatment of Chronic Pain. Ann Intern Med. 2015; 162(4):295-300. doi:10.7326/M14-2775

9. Català E, Ferrándiz M, Lorente L, Landaluce Z, Genové M. Uso de opioides en el tratamiento del dolor crónico no oncológico. Recomendaciones basadas en las guías de práctica clínica. Rev Esp Anestesiol Reanim. 2011; 58:283-289. doi:10.1016/S00349356(11)70063-9

10. Tormo Molina J, Marín Conde L, González Urbano M, Ruiz Pérez de la Blanca M, Robles Martín J, Vivar Simón M. Prescripción de opioides mayores en pacientes con dolor no oncológico: descripción de sus características en una zona de salud de atención primaria. Rev Soc Esp Dolor 2017; 24(1): 19-26.

11. Utilización de medicamentos opioides en España durante el periodo 2008-2015 [Monografía en Internet]. Madrid: Agencia Española del Medicamento y Productos Sanitarios; 2017 [acceso 1 de septiembre de 2018] Disponible en: https://www.aemps.gob. es/medicamentosUsoHumano/observatorio/docs/opioides-2008-2015.pdf

12. Doukkali A, Murillo D, Motilva V, Lacalle JR. Intervención farmacéutica y percepción del paciente sobre su dolor. Farmacéuticos Comunitarios 2011 Sep 30; 2(3): 93-99.

13. Casals M, Samper D. Epidemiology, prevalence and quality of life of non-malignant chronic pain. ITACA study. Rev Soc Esp Dolor 2004; 11: 260-269.

14. Noceda Bermejo JJ, Moret Ten C, Lauzirika Alonso I. Tratamiento del dolor osteomuscular crónico. Aten Primaria 2007; 39(1): 29-33. doi: $10.1157 / 13098276$

15. Gamero F, Gabriel R, Carbonell J, Tornero J, Sánchez I. Pain in Spanish rheumatology outpatient offices: EPIDOR epidemiological study. Rev Clin Esp. 2005; 205:157-63.

16. Rigueira García AI. Cumplimiento terapéutico: ¿qué conocemos de España? Aten Primaria 2001; 27(8): 559-68. doi:10.1016/S0212-6567(01)78860-9 